\title{
ANÁLISIS DE LA RELACIÓN HÁBITOS Y ACEPTACIÓN DE 9'S COMO ELEMENTO DE COMPETITIVIDAD EN LA PYME MANUFACTURERA DE AGUASCALIENTES, MÉXICO
}

Analysis of the relationship between habits and acceptance of the 9's as a competitiveness factor in manufacturing SMEs in Aguascalientes

\section{Octavio Hernández-Castorena (iD}

PhD en Ciencias Administrativas, Universidad Autónoma de Aguas Calientes, México, ohernandez@correo.uaa.mx

\section{Mónica Colín-Salgado}

PhD en Estudios de Género, Universidad Externado de Colombia, Bogotá-Colombia, colinsalgado@gmail.com

\author{
Noé Velásquez-Espinosa (iD \\ PhD en Ciencias de la Administración, Universidad Externado de Colombia, Bogotá-Colombia, \\ noe.velasquez@uexternado.edu.co
}

\begin{abstract}
Cómo referenciar / How to cite
Hernández-Castorena, O., Colín-Salgado, M. y Velásquez-Espinosa, N. (2018). Análisis de la relación hábitos y aceptación de 9's como elemento de competitividad en la pyme manufacturera de Aguascalientes, México. Revista CEA, 4(7), 29-47. https://doi.org/10.22430/24223182.757
\end{abstract}

Recibido: 25 de agosto de 2017

Aceptado: 30 de septiembre de 2017

\section{Resumen}

El presente estudio muestra en fase experimental, y con apoyo de un instrumento de evaluación, el análisis del desarrollo de hábitos y aceptación a partir de cada elemento de la teoría propuesta de Covey en cómo un trabajador de la pyme manufacturera acepta sin problemas la filosofía japonesa 9's. Para llevar a cabo el estudio se aplicó una encuesta a 8 empresas pyme del sector manufactura en Aguascalientes, México en el periodo febrero y abril del 2015, donde se eligieron de manera aleatoria a 200 trabajadores de un mismo nivel organizacional. El presente estudio tiene un enfoque cuantitativo, un método de correlación, con análisis descriptivos de media.
Además, se realizó un análisis de fiabilidad considerando alfa de Cronbach y análisis de regresión utilizando el software SPSS versión 19, resultando con este proceso que el personal de este tipo de empresas tiene tendencias a ser proactivo, sinérgico, prioritario, entiende las instrucciones a la primera vez, es analista y considera que en la relación ganar-ganar tiene más beneficio que efecto perjudicial para la dinámica operativa de la empresa.

Palabras clave: filosofía japonesa 9's, desarrollo de hábitos, pyme manufacturera, cultura organizacional. 


\section{Abstract}

This study presents the experimental stage of the analysis of habits and acceptance aided by an evaluation tool and based on each element of the theory proposed by Covey on how an employee at a manufacturing SME accepts the Japanese philosophy 9's without hassle. An interview was applied to 8 manufacturing SMEs in Aguascalientes, Mexico, from February to April 2015. Two hundred employees at the same organizational level were randomly selected. This work adopted a quantitative correlational approach that includes descriptive analyses of the mean. Additionally, the reliability was analyzed by considering Cronbach's alpha and a regression analysis using SPSS software version 19. The results suggest that the personnel at this type of companies tends to be proactive, synergic, can prioritize and understands instructions as soon as they are provided. They are also analytic and consider that a win-win situation is more beneficial than detrimental to the operational dynamics of the company.

Keywords: Japanese philosophy 9's, Development of habits, Manufacturing SMEs, Organizational Culture.

\section{INTRODUCCIÓN}

Desarrollar un hábito siempre ha sido una condición normal en el ser humano (Camic, 1989; Hodgson, 2007). Desde luego que en los grupos sociales es importante saber diferenciar un hábito de una costumbre, ya que las personas en su proyecto natural de desarrollo requieren de estrategias que les permitan contribuir en el desempeño productivo de las empresas que los contraten (Covey, 1998). En este sentido, para una empresa el tener personal interesado en desarrollar en particular hábitos, esto representa un acierto para su crecimiento organizacional interno, desde luego siempre y cuando el hábito sea positivo y con un sentido propio que le favorezca a la organización (Guedes, 2000). Esto significa que, al tener personal propositivo, es más sencillo adoptar sistemas o métodos de trabajo útiles con la finalidad de conseguir los fines que la empresa planifique según los requerimientos de sus clientes, de lo contrario, el tener personal interesado en evitar hacer equipos de trabajo, los resultados podrán ser desfavorables que afecten a los clientes mismos que no tendrían problema en cambiar de proveedor (Covey, 1998; Ortiz, Esquivel \& Hernández, 2016).

Considerando entonces que el desarrollo de hábitos es benéfico para las personas y por ende para las organizaciones, es importante resaltar que aprender nuevas metodologías será siempre para las personas más fácil de colaborar en el desarrollo de sus respectivas organizaciones (Hirano, 2000; Hernández, 2007), por lo que cualquier implementación por compleja que esta sea, teniendo como meta empresarial el crecimiento, desempeño (Prins \& Gómez, 2015), así como mejora constante, el tener empleados comprometidos, disciplinados y sinérgicos, será para la empresa una garantía de cumplir con los proyectos y metas que se tengan proyectados (Morgan, 1997; Kume, 1989). Bajo esta perspectiva, los gerentes tienen el reto de integrar a su personal en trabajar en equipo con la finalidad de evaluar qué sistema de trabajo es más efectivo que contribuya al desarrollo de la organización teniendo en cuenta el rechazo por parte del personal en aprovechar cualquier método de trabajo que se quiera implementar (Brown \& Lockett 2004; Vargas, 2004; Anda \& Rosales 2009). En este sentido, el uso y aprovechamiento de una herramienta de trabajo como la japonesa de 9 's requiere de previo conocimiento y de la metodología en cómo adaptar las 9's, con el propósito de evitar al máximo el rechazo por 
parte de los empleados (Hirano, 2000; Imai, 2000; Deming, 1989; 1982).

Por lo que dentro de las empresas manufactureras donde la dinámica operativa interna demanda controles precisos sobre los recursos materiales así como del cumplimiento de los procesos y aspectos de calidad, el integrar una herramienta de trabajo oriental como la japonesa 9's, permite que el mando de los recursos y su metodología facilita que los participantes (esperando que también sean directivos), adopten con mayor facilidad una herramienta de estas características donde se pondrá prueba a todos los miembros de las organizaciones, ya que en teoría es fácil su adopción pero en la práctica se requiere principalmente del desarrollo de hábitos y desaprender los hábitos a los que estén habituados los trabajadores (Hernández, 2007; De Anda \& Rosales 2009; Covey, 1998).

La filosofía japonesa 9's está integrada por nueve elementos: seiri, seiton, seiso, seiketsu, shitsuke, shikari, shitsukoku, seisho y seido (español: orden, clasificación, limpieza, disciplina, educación, constancia, compromiso, coordinación y estandarización), que se pueden ir desarrollando según sea la pertinencia y urgencia de la empresa por esta adopción (Hirano, 2000; Aguilera, Gonzáles \& Hernández, 2013; Hernández, López \& Macías, 2012). El presente trabajo de investigación tiene el propósito de discutir sobre cuál elemento de los integrados en el bloque de hábitos incide de forma positiva en como los trabajadores aceptan sin problema la filosofía japonesa 9's y con ello generar propuestas de mejora para el caso en que existan elementos que afecten en la implementación de esta importante y productiva herramienta de trabajo.

\section{MARCO TEÓRICO}

\section{Hábitos}

Los hábitos son fenómenos que experimenta el ser humano por naturaleza y estos se manifiestan a partir de la repetición de la misma actividad en un determinado período para establecerse por un tiempo en el que podemos llamar de rutina, desde luego que pueden ser clasificados como buenos o malos (Covey, 1998). Acorde a Lorenz (2000), es importante diferenciar un hábito de una costumbre, en este sentido, refiere el resultado de una serie de actividades realizadas en una frecuencia de tiempo constante y con sentido de conciencia por parte del individuo, donde evidentemente tiene el propósito de llegar a un fin; por otro lado, una costumbre es el resultado de actos repetidos no exactamente con la misma intención consciente por parte del individuo y que obedece en parte a actos sociales o de grupo, como lo refiere Guedes (2000).

Es cierto que los individuos, los cuales por naturaleza están inmersos en participaciones diversas en la sociedad, no pueden dejar a un lado el pertenecer a grupos de actividades cualquiera que esta sea, ya sea de trabajo, social o diversión, al margen de casos especiales donde llevan sus dinámicas sociales de manera personal (pero dentro de una sociedad), y es aquí donde toman patrones sociales y se sesgan en adoptar así como generar hábitos con fines involuntarios, pero con una meta específica (Beuren \& Macohon, 2011). Esto quiere decir que un individuo no puede dejar de influenciarse ante las rutinas sociales que se manejen en su entorno donde acostumbra llevar su vida personal o laboral, lo que evidentemente lo lleva en adoptar hábitos sociales que adapta o ajusta a su particular beneficio personal (Guerreiro, Frezatti \& Casado, 2004). 
Por otro lado, Scapens (1994) menciona que los hábitos tienen tendencias a ser generados por las instituciones, es decir, una conducta es adoptada por un individuo en gran medida porque las instituciones en general tienen los sistemas o métodos para incidir en las personas, con cierta garantía, la adopción de un hábito. Así mismo, Beuren y Macohon, (2011), dimensionan que es más institucional u organizacional, ya que estas tienen en su sistema de trabajo reglas definidas, procedimientos y políticas afines a la naturaleza de cada institución u organización mientras que una rutina tiene más tendencia a ser una formalidad social que se adopta derivado de las costumbres que se tengan por regiones o sistemas. Lo cierto es que las actividades institucionales $u$ organizacionales suelen ser más constantes y duraderas (Oliver, 1997).

En este sentido, para las personas en general y al margen de las tendencias culturales que se tengan, cuando los individuos tienen influencia en tomar actitudes o costumbres que se estén desarrollando por grupos en el aspecto social u organizacional, es importante evaluar si estos hábitos podrán resultar de impacto positivo o negativo puesto que en ambos sentidos habrá en las personas nuevas formas de conducirse y esto tendrá por supuesto impacto en el espacio en el que se desenvuelvan y desde luego que para el caso de una actividad empresarial, esta integración de los trabajadores tendrá sus consecuencias favorables o desfavorables acorde a las políticas y normas de cada empresa (Caez \& Casas, 2007; Cuéllar, 2007). Es importante que cada individuo, una vez que sea influenciado por la adopción de un hábito, identifique el efecto e impacto de este nuevo modelo de actuación (Covey, 1998).

Por otro lado y analizando a mayor profundidad lo que representa un hábito, ya sea como desarrollo o integración en las personas, Mc Phail (2012), considera, citando una referencia teórica de Claude Hopkins (1934), que los individuos tienen tendencias en aceptar que las acciones personales que se trabajan diariamente dentro de un ambiente social, son el resultado de su propia voluntad y razón humana, lo que a su vez es considerado como un producto de hábitos que se cultivan de manera constante y consciente con el enfoque de llevar una vida más manejable. Esto permite afirmar que cuando un individuo tiene el interés particular de manejarse dentro de un ambiente con plena conciencia de pertenecer a un grupo determinado, puede generar hábitos mismos que se implementarán sin problema siempre y cuando sea de manera constante, ya que el individuo le ve sentido en repetir la misma actividad en un espacio idéntico que a la postre le permitirá generar un nuevo hábito (Duhigg, 2012).

En este sentido, un hábito desarrolla un poder tal, que una vez integrado en la vida diaria de un individuo cuesta trabajo eliminarlo de inmediato (Duhigg, 2012). Sin embargo, es importante resaltar que esta condición disciplinar se implementará en las acciones diarias de un individuo, siempre y cuando el propio personaje tenga interés en su adopción o en dejar que las cosas sucedan de manera natural (Aymerich, 2010). Asimismo, es importante recordar que los individuos por naturaleza dejan que en sus decisiones o integración a nuevas formas de vida incidan sus emociones y aspectos de carácter humanitario (Dichter, 1960, 1964). Los hábitos pasan por etapas ya analizadas con anterioridad, en las cuales los individuos inconscientemente analizan su proceso, entorno y alcances generando con ello nuevas formas de adaptarse a las exigencias sociales y en particular laborables (Covey, 1998).

\section{Filosofía japonesa 9's}

Para las empresas en general, en la actualidad, es necesario integrar en sus actividades 
operativas herramientas y sistemas que les permitan además de optimizar sus procesos garantizar la calidad de los productos o servicios que estas ofrezcan al consumidor, y para ello requiere de dos cosas importantes: por un lado, tener procesos definidos, confiables y bien administrados; y por otro lado, tener un recurso humano preparado y capaz de cumplir con los objetivos que estén definidos de manera organizacional (Deming, 1982; Aguilera, González \& Hernández, 2013; Hernández, Colín \& Galindo, 2016).

En este sentido, para que dentro de una organización se puedan armonizar procesos administrativos y personal capacitado se requiere de liderazgo y sistemas de capacitación, acorde a las necesidades de cada empresa, en el sentido de administrar y de visualizar productos o servicios de calidad (Cantú, 2006; Deming, 1982). Dentro de esta armonía de desarrollo y crecimiento, el personal requiere de madurez para adoptar sistemas o métodos de trabajo que les permita mejorar sus actividades internas, y para ello, es necesario que el líder tenga la visión de anticiparse a las necesidades de la empresa para que las propuestas puedan ser aceptadas sin problemas siempre y cuando sus hábitos de trabajo sean positivos y sus costumbres naturales sean el de innovar así como el de mejorar tanto procesos como sistemas de trabajo (Deming, 1982; Pérez, 2011).

Entre las herramientas diversas de clase mundial que se conocen a nivel empresarial y que son útiles para la mejora de cualquier actividad interna de las organizaciones está la filosofía japonesa 9's, herramienta que desde 1945 ha sido probada con éxito, primero bajo el concepto de 5's con resultados incidentes en aspectos de productividad, seguridad en el control de los materiales, control de costos y control de la calidad de productos, así como de procesos (Hernández, et al., 2016). Esta herramienta de origen oriental tiene diversos propósitos una vez implementada en las empresas, siempre y cuando se integre de manera correcta con el personal idóneo, además cabe señalar que esta filosofía puede desarrollarse en cualquier organización sin importar el sector y giro a que pertenezcan (Hernández, 2007; Aguilera, González \& Hernández, 2013).

La filosofía japonesa 9's está integrada por 9 secciones, las cuales en orden de implementación se muestran a continuación (Hirano, 2000; Baltazar, 2005; Hernández, 2007; Hernández, Colín, \& Galindo, 2016):

SEIRI - Orden; SEITON - Clasificación; SEISO Limpieza y pulcritud; SEIKETSU - Educación; SHITSUKE - Disciplina; SHIKARI - Constancia; SHITSUKOKU - Compromiso; SEISHOO Coordinación; y SEIDO - Estandarización.

El presente estudio tiene como propósito analizar cómo los hábitos, en cada una de los factores propuestos en el modelo teórico, incide en los trabajadores para que acepten con facilidad la filosofía japonesa 9's (Covey, 2012; Aguilera, González \& Hernández, 2012) en este sentido, se plantean las siguientes hipótesis:

H1: a mayor desarrollo de hábitos en el trabajador, mejor facilidad de implementación de la filosofía japonesa 9's en la pyme Manufacturera de Aguascalientes, en México.

\section{METODOLOGÍA}

En el presente estudio, se analiza la situación actual sobre los hábitos de los trabajadores y la manera en cómo relacionan esta actitud en su interés por aceptar la filosofía japonesa 9's, disciplina de origen oriental cuyo propósito es incidir de manera personal en el trabajador para que mejore su desempeño dentro de la organización en la cual presta sus servicios en 
empresas pyme manufactureras. Para ello realizando previa consulta en la plataforma del Institutito Nacional de Estadística y Geografía (DENUE, 2015) en el estado de Aguascalientes, se obtiene un registro de 442 empresas a las cuales se evaluó de manera directa por parte del gerente de cada organización el tipo de hábitos que tienen sus empleados electos de manera aleatoria. Es importante señalar que el estudio se llevó a cabo con empresas que facilitaron la realización del trabajo de campo donde al menos hubiera 25 trabajadores en el área operativa, dando un total general de 200 empleados. La presente investigación es de enfoque cuantitativo de corte longitudinal, con un método analítico y descriptivo. EI instrumento de medición está en su fase experimental y es por ello que está a discusión los resultados estadísticos en particular los análisis de fiabilidad, Anova y regresión lineal con el propósito de mejorar en futuras líneas de investigación el instrumento aplicado en el presente estudio.

\section{Desarrollo de medidas}

Para el presente estudio se utilizó un instrumento de evaluación integrado por 2 bloques, los cuales se midieron con escala Likert de 1-5, con operacionalización desde total desacuerdo hasta un total acuerdo. El primer bloque denominado «hábito de los trabajadores» está compuesto por 7 dimensiones, el cual se muestra en la Tabla 1: (Covey, 2012). El segundo bloque está identificado como «Aceptación de la filosofía japonesa 9's en las áreas de trabajo» (Aguilera, González \& Hernández, 2012), el cual se muestra en la Tabla 2. Es importante resaltar que actualmente el instrumento está en su fase experimental.

En la Figura 1 se muestra el modelo teórico aplicado a la presente investigación, el cual muestra la relación de la hipótesis que existe entre los bloques principales de «Hábitos de los trabajadores» (la cual integra 7 dimensiones adaptada de Covey, 2012) y «Aceptación de la filosofía japonesa 9's» en las áreas de trabajo.

Respecto a los análisis de fiabilidad del instrumento aplicado a los trabajadores de las 8 empresas pyme manufactureras, la Tabla 3 muestra los resultados de fiabilidad bajo el uso del alfa de Cronbach, el cual permite la aceptación de valores a partir de 0.7 (Frías, 2014). Sin embargo, en fase experimental o primeras fases del análisis, es permitido continuar con el estudio al margen de la referencia que hace George \& Mallery (2003) y Nunnally (1967) respecto a los valores de 0.6 (análisis cuestionable) y 0.5 (valores pobres).

Los resultados descritos en Tabla 3 muestran que las dimensiones de "reflexivo» y "afilar la sierra» tiene valores bajos o que demanda un análisis más a detalle sobre la intención de la escala y de la aplicación en esta sección de la encuesta por parte del evaluador para con el objeto de estudio. En la Tabla 4 se muestran los valores descriptivos de cada una de las dimensiones. 
Tabla 1. Bloque 1 - identificado como «Hábitos de los trabajadores»

Table 1. Category 1 - Workers' habits

\begin{tabular}{|c|c|c|}
\hline Variable & & Descripción de la variable \\
\hline \multicolumn{3}{|c|}{ En esta sección se mide el hábito de PROACTIVIDAD: } \\
\hline \multirow{5}{*}{ Proactivo } & P01 & El trabajador es dinámico y participativo \\
\hline & P02 & El trabajador cumple con los compromisos en tiempo y forma \\
\hline & P03 & El trabajador requiere poca supervisión \\
\hline & P04 & El trabajador ante una emergencia motiva a sus compañeros \\
\hline & P05 & El trabajador tiene iniciativa \\
\hline \multirow{5}{*}{ Reactivo } & PR1 & El trabajador es productivo si existe presión en la supervisión \\
\hline & PR2 & El trabajador con frecuencia se queja del trabajo \\
\hline & PR3 & El trabajador con frecuencia no cumple con sus tareas en tiempo \\
\hline & PR4 & El trabajador acostumbra evitar trabajar en equipo si hay tareas encomendadas \\
\hline & PR5 & El trabajador acostumbra desestabilizar el ambiente de trabajo \\
\hline \multicolumn{3}{|c|}{ En esta sección se mide el hábito de TENER UN FIN EN MENTE: } \\
\hline FM1 & \multicolumn{2}{|c|}{ El trabajador organiza su agenda al iniciar el turno laboral } \\
\hline FM2 & \multicolumn{2}{|r|}{ El trabajador da seguimiento durante la jornada a su plan de trabajo } \\
\hline FM3 & \multicolumn{2}{|r|}{ El trabajador planea por sí mismo el uso de recursos alternos para cumplir su trabajo } \\
\hline FM4 & \multicolumn{2}{|r|}{ El trabajador considera siempre optimizar los recursos de la empresa } \\
\hline FM5 & \multicolumn{2}{|c|}{ El trabajador acostumbra escribir su plan de trabajo } \\
\hline FM6 & \multicolumn{2}{|r|}{ El trabajador entiende a la primera las instrucciones y las ejecuta } \\
\hline FM7 & \multicolumn{2}{|r|}{ El trabajador es consciente de sus fortalezas y propone nuevas ideas } \\
\hline FM8 & \multicolumn{2}{|r|}{ El trabajador se apoya en sus compañeros para cumplir con las metas de trabajo } \\
\hline \multicolumn{3}{|c|}{ En esta sección se mide el hábito de PRIMERO LO PRIMERO: } \\
\hline \multirow{4}{*}{ C1 Moroso } & PM1 & El trabajador generalmente no prioriza sus actividades \\
\hline & PM2 & El trabajador cumple tarde con sus tareas \\
\hline & PM3 & El trabajador prepara sus actividades a última hora \\
\hline & PM4 & El trabajador programa actividades no importantes \\
\hline \multirow{4}{*}{ C2 Prioritario } & PP1 & El trabajador tiene la capacidad de establecer prioridades \\
\hline & PP2 & $\begin{array}{l}\text { El trabajador tiene la capacidad de programar sus actividades laborales acorde a } \\
\text { la prioridad }\end{array}$ \\
\hline & PP3 & El trabajador tiene la capacidad de tener sus tareas bajo control \\
\hline & PP4 & El trabajador tiene más de 2 actividades extra laborales \\
\hline \multirow{4}{*}{ C3 Sumiso } & PS1 & El trabajador se compromete al mismo tiempo con muchas tareas \\
\hline & PS2 & El trabajador por lo regular queda mal con un compromiso \\
\hline & PS3 & El trabajador por lo regular no es disciplinado \\
\hline & PS4 & El trabajador se deja influir por otras tareas no importantes \\
\hline \multirow{4}{*}{ C4 Flojo } & PF1 & El trabajador generalmente pierde el tiempo en cosas ajenas al trabajo \\
\hline & PF2 & El trabajador generalmente busca pretextos para evadir una responsabilidad \\
\hline & PF3 & El trabajador generalmente no cumple al $100 \%$ con la efectividad de sus tareas \\
\hline & PF4 & El trabajador generalmente llega tarde a sus compromisos laborales \\
\hline \multicolumn{3}{|c|}{ En esta sección se mide el hábito de GANAR - GANAR: } \\
\hline \multirow{4}{*}{$\begin{array}{l}\text { Ganar - } \\
\text { Perder }\end{array}$} & GP1 & $\begin{array}{l}\text { El trabajador en una negociación acostumbra tener el principal beneficio con el } \\
\text { jefe }\end{array}$ \\
\hline & GP2 & $\begin{array}{l}\text { El trabajador en una negociación acostumbra tener el principal beneficio con sus } \\
\text { compañeros }\end{array}$ \\
\hline & GP3 & $\begin{array}{l}\text { El trabajador en una negociación acostumbra tener el principal beneficio con la } \\
\text { empresa }\end{array}$ \\
\hline & GP4 & El trabajador suele sentirse superior al resto de los demás trabajadores \\
\hline
\end{tabular}




\begin{tabular}{|c|c|c|}
\hline \multirow{4}{*}{$\begin{array}{l}\text { Perder - } \\
\text { Ganar }\end{array}$} & PG1 & El trabajador generalmente cede ante la presión del jefe en un acuerdo \\
\hline & PG2 & El trabajador generalmente cede ante la presión de sus compañeros \\
\hline & PG3 & El trabajador generalmente cede ante la presión de los intereses de la empresa \\
\hline & PG4 & $\begin{array}{l}\text { El trabajador generalmente es tímido actitudinalmente comparado con el resto } \\
\text { de sus compañeros }\end{array}$ \\
\hline \multirow{4}{*}{$\begin{array}{l}\text { Perder - } \\
\text { Perder }\end{array}$} & PE1 & El trabajador ante una desventaja suele convidar del problema a los compañeros \\
\hline & PE2 & El trabajador busca la manera de afectar los intereses de compañeros \\
\hline & PE3 & El trabajador busca la manera de afectar el cumplimiento de las metas \\
\hline & PE4 & $\begin{array}{l}\text { El trabajador en una relación de trabajo si no tiene beneficio busca que otros no } \\
\text { lo tengan }\end{array}$ \\
\hline \multirow{4}{*}{ Ganar - Ganar } & GG1 & El trabajador sabe llegar a un acuerdo donde ganen ambas partes \\
\hline & GG2 & El trabajador comparte su conocimiento con el resto de sus compañeros \\
\hline & GG3 & El trabajador busca la manera de generar mejoras en su trabajo \\
\hline & GG4 & El trabajador busca la manera de capacitarse en bien de su actividad \\
\hline \multicolumn{3}{|c|}{ En esta sección se mide el hábito de PRIMERO ENTENDER Y LUEGO SER ENTENDIDO: } \\
\hline EE1 & \multicolumn{2}{|r|}{ El trabajador generalmente esta distraído al momento de que se le da una instrucción } \\
\hline EE2 & \multicolumn{2}{|r|}{$\begin{array}{l}\text { El trabajador generalmente durante una instrucción de trabajo finge que está poniendo } \\
\text { atención }\end{array}$} \\
\hline EE3 & \multicolumn{2}{|r|}{ El trabajador generalmente entiende la instrucción según su estado de ánimo } \\
\hline EE4 & \multicolumn{2}{|r|}{ El trabajador generalmente entiende la instrucción pero no el sentido de la instrucción } \\
\hline EE5 & \multicolumn{2}{|r|}{ El trabajador generalmente entiende la instrucción según su propia conveniencia } \\
\hline \multicolumn{3}{|c|}{ En esta sección se mide el hábito de SINERGIZAR: } \\
\hline \multirow{3}{*}{ Reflexivo } & SR1 & El trabajador es menos emotivo al tomar decisiones \\
\hline & SR2 & El trabajador pone atención a los detalles de una instrucción \\
\hline & SR3 & El trabajador no se precipita ante una instrucción \\
\hline \multirow{3}{*}{ Independiente } & SI1 & El trabajador comparte y delega éticamente una responsabilidad \\
\hline & $\mathrm{SI} 2$ & El trabajador acepta las ideas de los compañeros \\
\hline & $\mathrm{SI3}$ & $\begin{array}{l}\text { El trabajador generalmente es promotor de mejoras para apoyo de su área y } \\
\text { otras }\end{array}$ \\
\hline \multirow{3}{*}{ Planeador } & SP1 & El trabajador expresa frecuentemente su opinión cuando se asigna una tarea \\
\hline & SP2 & El trabajador requiere de los puntos de vista de los demás \\
\hline & SP3 & $\begin{array}{l}\text { El trabajador suele ser menos rígido con el apoyo en una tarea con los } \\
\text { compañeros }\end{array}$ \\
\hline \multirow{3}{*}{ Analista } & SA1 & El trabajador acepta fácilmente sus errores \\
\hline & SA2 & El trabajador considera el uso de alternativas para la solución de problemas \\
\hline & SA3 & $\begin{array}{l}\text { El trabajador considera los sentimientos de los demás al momento de tomar una } \\
\text { decisión de trabajo }\end{array}$ \\
\hline \multicolumn{3}{|c|}{ En esta sección se mide el hábito de AFILAR LA SIERRA: } \\
\hline AS1 & \multicolumn{2}{|r|}{ El trabajador utiliza los descansos para analizar un problema laboral } \\
\hline AS2 & \multicolumn{2}{|r|}{$\begin{array}{l}\text { El trabajador utiliza los descansos para distraerse en actividades alteras a la actividad de } \\
\text { trabajo }\end{array}$} \\
\hline AS3 & \multicolumn{2}{|r|}{ El trabajador generalmente pide apoyo ante la demora en la solución de un problema } \\
\hline AS4 & \multicolumn{2}{|r|}{ El trabajador generalmente se documenta ante la demora en la solución de un problema } \\
\hline AS5 & \multicolumn{2}{|r|}{$\begin{array}{l}\text { El trabajador ante un problema, deja momentáneamente su actividad y realiza } \\
\text { actividades diferentes. }\end{array}$} \\
\hline
\end{tabular}
Fuente: Hernández, Colín y Galindo (2016). 
Tabla 2. Bloque 2 - identificado como Aceptación de la Filosofía Japonesa 9's en las áreas de trabajo. Table 2. Category 2 - Acceptance of Japanese Philosophy 9's in work areas

\begin{tabular}{l|l}
\hline Variable & \multicolumn{1}{c}{ Descripción de la variable } \\
\hline AFJ1 & El trabajador no acepta por ningún motivo la adopción de 9's \\
\hline AFJ2 & El trabajador no ve beneficios al integrar 9's \\
\hline AFJ3 & El trabajador tiene escaso interés en adoptar las 9's \\
\hline AFJ4 & El trabajador tiene escaso conocimiento sobre la teoría 9's \\
\hline AFJ5 & El trabajador cuestiona la eficiencia de la teoría 9's \\
\hline AFJ6 & El trabajador muestra interés por conocer la funcionabilidad de 9's \\
\hline AFJ7 & $\begin{array}{l}\text { El trabajador encuentra poco impacto de uso 9's en el desempeño de sus } \\
\text { funciones }\end{array}$ \\
\hline
\end{tabular}

Fuente: Hernández, Colín y Galindo (2016).

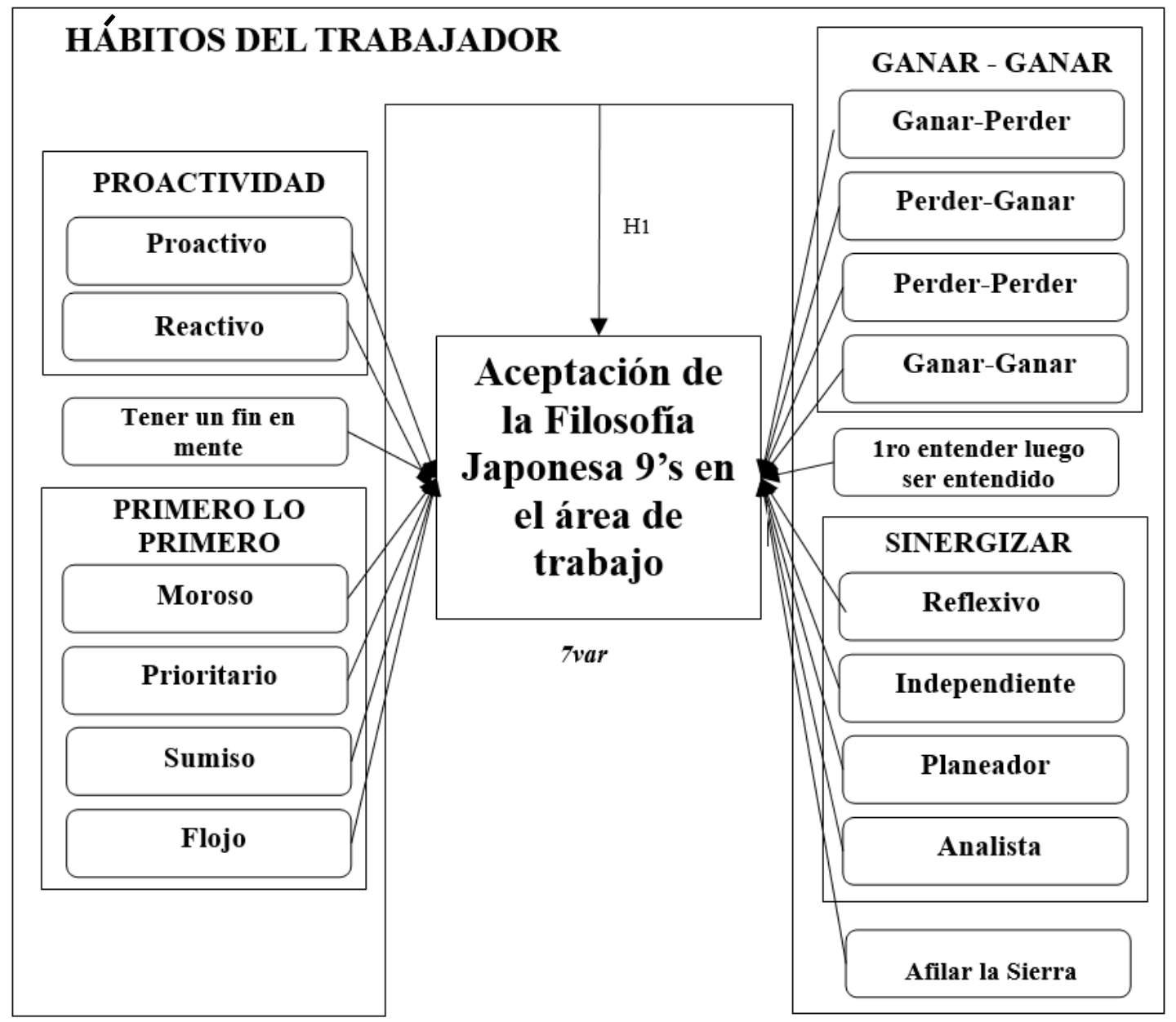

Figura 1. Modelo Teórico de la Investigación

Figure 1. Theoretical research model

Fuente: elaboración propia adaptado de Covey, 2012; Aguilera, González y Hernández, 2012. 
Tabla 3. Análisis de fiabilidad con Alfa de Cronbach de las dimensiones de los bloques «Hábitos de los trabajadores y aceptación de la filosofía japonesa 9's en el área de trabajo»

Table 3. Reliability analysis of the dimensions of Categories Workers' habits and Acceptance of the Japanese Philosophy 9's in work areas with Cronbach's Alpha

\begin{tabular}{|c|c|c|c|c|c|}
\hline Bloque & Factor & Dimensión & Variables & Cantidad & $\begin{array}{c}\text { Alfa de } \\
\text { Cronbach }\end{array}$ \\
\hline \multirow{17}{*}{$\begin{array}{l}\text { HÁBITOS DE LOS } \\
\text { TRABAJADORES }\end{array}$} & \multirow[t]{2}{*}{ Proactividad } & Proactivo & P01 - P05 & 5 & 0.774 \\
\hline & & Reactivo & PR1 - PR5 & 5 & 0.781 \\
\hline & $\begin{array}{c}\text { Tener un fin en } \\
\text { mente }\end{array}$ & $\begin{array}{l}\text { Tener un fin en } \\
\text { mente }\end{array}$ & FM1 - FM8 & 8 & 0.815 \\
\hline & \multirow{4}{*}{$\begin{array}{l}\text { Primero lo } \\
\text { Primero }\end{array}$} & Moroso & PM1 - PM4 & 4 & 0.909 \\
\hline & & Prioritario & PP1 - PP4 & 4 & 0.676 \\
\hline & & Sumiso & PS1 - PS4 & 4 & 0.772 \\
\hline & & Flojo & PF1 - PF4 & 4 & 0.912 \\
\hline & \multirow{4}{*}{ Ganar - Ganar } & Ganar-Perder & GP1 - GP4 & 4 & 0.712 \\
\hline & & Perder-Ganar & PG1 - PG4 & 4 & 0.708 \\
\hline & & Perder - Perder & PE1 - PE4 & 4 & 0.845 \\
\hline & & Ganar-Ganar & GG1 - GG4 & 4 & 0.787 \\
\hline & $\begin{array}{c}\text { Primero } \\
\text { entender y } \\
\text { luego ser } \\
\text { entendido }\end{array}$ & $\begin{array}{l}\text { Primero Entender } \\
\text { y luego ser } \\
\text { entendido }\end{array}$ & EE1 - EE5 & 5 & 0.906 \\
\hline & \multirow{4}{*}{ Sinergizar } & Reflexivo & SR1 - SR3 & 3 & 0.236 \\
\hline & & Independiente & $\mathrm{SI} 1-\mathrm{SI} 3$ & 3 & 0.645 \\
\hline & & Planeador & SP1 - SP3 & 3 & 0.523 \\
\hline & & Analista & SA1 - SA3 & 3 & 0.583 \\
\hline & Afilar la sierra & Afilar la sierra & AS1 - AS5 & 5 & 0.499 \\
\hline \multicolumn{3}{|c|}{ Aceptación de la Filosofía Japonesa 9’s en el área de trabajo } & AFJ1 - AFJ7 & 7 & 0.806 \\
\hline
\end{tabular}

Fuente: elaboración propia. 
Tabla 4. Análisis descriptivos

Table 4. Descriptive analysis

\begin{tabular}{|c|c|c|c|c|}
\hline Bloque & Factor & Dimensión & Variable & Media \\
\hline \multirow[t]{17}{*}{$\begin{array}{l}\text { Hábitos de los } \\
\text { Trabajadores }\end{array}$} & \multirow[t]{2}{*}{ Proactividad } & Proactivo & $\begin{array}{l}\text { El trabajador cumple con los } \\
\text { compromisos en tiempo y forma }\end{array}$ & 4.04 \\
\hline & & Reactivo & $\begin{array}{l}\text { El trabajador es productivo si existe } \\
\text { presión en la supervisión }\end{array}$ & 3.64 \\
\hline & \multicolumn{2}{|c|}{ Tener un fin en mente } & $\begin{array}{l}\text { El trabajador entiende a la primera } \\
\text { las instrucciones y las ejecuta }\end{array}$ & 4.00 \\
\hline & \multirow{4}{*}{$\begin{array}{l}\text { Primero lo } \\
\text { Primero }\end{array}$} & Moroso & $\begin{array}{l}\text { El trabajador programa actividades } \\
\text { no importantes }\end{array}$ & 2.68 \\
\hline & & Prioritario & $\begin{array}{l}\text { El trabajador tiene la capacidad de } \\
\text { programar sus actividades } \\
\text { laborales acorde a la prioridad }\end{array}$ & 4.09 \\
\hline & & Sumiso & $\begin{array}{l}\text { El trabajador se compromete al } \\
\text { mismo tiempo con muchas tareas }\end{array}$ & 3.03 \\
\hline & & Flojo & $\begin{array}{l}\text { El trabajador generalmente pierde } \\
\text { el tiempo en cosas ajenas al trabajo }\end{array}$ & 2.73 \\
\hline & \multirow{4}{*}{ Ganar - Ganar } & Ganar-Perder & $\begin{array}{l}\text { El trabajador en una negociación } \\
\text { acostumbra tener el principal } \\
\text { beneficio con la empresa }\end{array}$ & 3.39 \\
\hline & & Perder-Ganar & $\begin{array}{l}\text { El trabajador generalmente cede } \\
\text { ante la presión de los intereses de } \\
\text { la empresa }\end{array}$ & 3.39 \\
\hline & & Perder - Perder & $\begin{array}{l}\text { El trabajador ante una desventaja } \\
\text { suele convidar del problema a los } \\
\text { compañeros }\end{array}$ & 2.82 \\
\hline & & Ganar-Ganar & $\begin{array}{l}\text { El trabajador busca la manera de } \\
\text { generar mejoras en su trabajo }\end{array}$ & 3.98 \\
\hline & \multicolumn{2}{|c|}{$\begin{array}{l}\text { Primero entender y luego ser } \\
\text { entendido }\end{array}$} & $\begin{array}{l}\text { El trabajador generalmente } \\
\text { entiende la instrucción según su } \\
\text { estado de ánimo }\end{array}$ & 2.60 \\
\hline & \multirow{4}{*}{ Sinergizar } & Reflexivo & $\begin{array}{l}\text { El trabajador pone atención a los } \\
\text { detalles de una instrucción }\end{array}$ & 3.83 \\
\hline & & Independiente & $\begin{array}{l}\text { El trabajador acepta las ideas de los } \\
\text { compañeros }\end{array}$ & 3.80 \\
\hline & & Planeador & $\begin{array}{l}\text { El trabajador expresa } \\
\text { frecuentemente su opinión cuando } \\
\text { se asigna una tarea }\end{array}$ & 3.61 \\
\hline & & Analista & $\begin{array}{l}\text { El trabajador considera el uso de } \\
\text { alternativas para la solución de } \\
\text { problemas }\end{array}$ & 3.90 \\
\hline & \multicolumn{2}{|c|}{ Afilar la sierra } & $\begin{array}{l}\text { El trabajador generalmente pide } \\
\text { apoyo ante la demora en la } \\
\text { solución de un problema }\end{array}$ & 3.96 \\
\hline \multicolumn{3}{|c|}{$\begin{array}{l}\text { Aceptación de la filosofía japonesa 9's en el área de } \\
\text { trabajo }\end{array}$} & $\begin{array}{l}\text { El trabajador muestra interés por } \\
\text { conocer la funcionabilidad de 9's }\end{array}$ & 3.38 \\
\hline
\end{tabular}


Respecto a los resultados mostrados en la Tabla 4, el desarrollo de hábitos en los trabajadores de la pyme manufacturera en Aguascalientes, México, muestran los siguientes efectos: los empleados tienen características de ser proactivos, suelen entender las instrucciones a la primera explicación, sin embargo, tienen tendencia a dejarse influenciar por su estado emocional. También tienen habilidad para clasificar las actividades prioritarias en una agenda de trabajo donde requieran cumplir con diversas tareas. Así mismo, son personas que prefieren en una negociación tener los mismos beneficios o entrar al esquema de ganar-ganar.

En las actividades operativas dentro de la empresa, los trabajadores generalmente son analistas con su propio trabajo, siendo con ello precavidos ante la inminente demora en la resolución de un problema en donde ellos prefieren pedir apoyo de un compañero de trabajo. Los resultados obtenidos acorde a la propuesta de Covey (2012), permiten afirmar que las empresas pyme manufactureras integran en su plantilla laboral empleados que no tienen problemas de disciplina ni de integración, como equipos sinérgicos de trabajo, por lo que podrán desarrollar sin problema una filosofía de trabajo, como lo son las 9's, con el propósito de mejorar la calidad de vida tanto del trabajador como de las operaciones internas de estas organizaciones (Hernández, 2007).

\section{RESULTADOS}

A continuación, en el presente apartado se muestran los resultados de análisis que permiten conocer la prueba de hipótesis, correlación entre dimensiones y efectos de regresión entre los mismos a través del tratamiento de datos en el software SPSS versión 19. En el caso particular de la Tabla 5, se muestran los resultados de la prueba estadística de Anova cuyo propósito es analizar si cada dimensión tiene significancia aprobatoria con respecto a la variable dependiente identificada como aceptación de la filosofía japonesa 9's en el área de trabajo.

Tabla 5. Análisis Anova del Modelo Teórico

Table 5. ANOVA analysis of the theoretical model

\begin{tabular}{l|c|c|c|c}
\hline \multicolumn{1}{c|}{ Dimensión } & Suma de cuadrados & $\mathrm{gl}$ & Valor de $\mathrm{F}$ & Sig. \\
\hline Proactivo & 20.815 & 26 & 1.787 & .015 \\
\hline Reactivo & 86.637 & 26 & 6.321 & .000 \\
\hline Tener un fin en mente & 21.565 & 26 & 1.644 & .033 \\
\hline Moroso & 143.620 & 26 & 6.282 & .000 \\
\hline Prioritario & 17.335 & 26 & 1.232 & .215 \\
\hline Sumiso & 101.626 & 26 & 6.302 & .000 \\
\hline Flojo & 149.865 & 26 & 6.139 & .000 \\
\hline Ganar - Perder & 64.536 & 26 & 4.735 & .000 \\
\hline Perder - Ganar & 68.476 & 26 & 5.481 & .000 \\
\hline Perder - Perder & 184.448 & 26 & 11.813 & .000 \\
\hline Ganar - Ganar & 19.785 & 26 & 1.119 & .324 \\
\hline Primero entender y luego ser entendido & 140.615 & 26 & 6.937 & .000 \\
\hline Reflexivo & 25.721 & 26 & 2.708 & .000 \\
\hline Independiente & 28.373 & 26 & 2.207 & .001 \\
\hline Planeador & 43.810 & 26 & 3.178 & .000 \\
\hline Analista & 39.317 & 26 & 2.571 & .000 \\
\hline Afilar la sierra & 36.185 & 26 & 4.840 & .000 \\
\hline
\end{tabular}

Fuente: elaboración propia a partir de los datos obtenidos del SPSS v21. 
Tabla 6. Resultados de la correlación de Pearson

Table 6. Results of the Pearson correlation

\begin{tabular}{|c|c|c|}
\hline Variable & $\begin{array}{c}\text { Aceptación de la filosofía japonesa 9's en el } \\
\text { área de trabajo } \\
\qquad\left({ }^{* * *} p<0.001 ;{ }^{* *} p<0.050\right)\end{array}$ & Resultados \\
\hline Proactivo & $\begin{array}{l}\text { ( - ) } 0.139 \\
\text { Valor de t }(-1.974) \\
\end{array}$ & $\begin{array}{c}R^{2} \text { ajustada }=0.014 \\
\quad F=3.989 \\
\text { FIV = 1.000; Sig. } 0.050\end{array}$ \\
\hline Reactivo & $\begin{array}{c}0.497^{* *} \\
\text { Valor de t (8.062) } \\
\end{array}$ & $\begin{array}{l}R^{2} \text { ajustada }=0.243 \\
\quad F=65.001 \\
\text { FIV = 1.000; Sig. } 0.000\end{array}$ \\
\hline Tener un fin en mente & $\begin{array}{c}0.090 \\
\text { Sin Valor de } t\end{array}$ & Sin Valor \\
\hline Moroso & $\begin{array}{c}0.504^{* *} \\
\text { Valor de t (8.215) } \\
\end{array}$ & $\begin{array}{l}R^{2} \text { ajustada }=0.250 \\
\quad F=67.483 \\
\text { FIV = 1.000; Sig. } 0.000\end{array}$ \\
\hline Prioritario & $\begin{array}{c}0.046 \\
\text { Sin Valor de t }\end{array}$ & Sin Valor \\
\hline Sumiso & $\begin{array}{c}0.547^{* *} \\
\text { Valor de t }(9.205) \\
\end{array}$ & $\begin{array}{l}R^{2} \text { ajustada }=0.296 \\
\quad F=84.732 \\
\text { FIV = 1.000; Sig. } 0.000\end{array}$ \\
\hline Flojo & $\begin{array}{c}0.547^{* *} \\
\text { Valor de t }(9.188)\end{array}$ & $\begin{array}{l}R^{2} \text { ajustada }=0.295 \\
\quad F=84.428 \\
\text { FIV = 1.000; Sig. } 0.000\end{array}$ \\
\hline Ganar-Perder & $\begin{array}{c}0.424^{* *} \\
\text { Valor de t (6.595) }\end{array}$ & $\begin{array}{l}R^{2} \text { ajustada }=0.176 \\
\quad F=43.490 \\
\text { FIV = 1.000; Sig. } 0.000\end{array}$ \\
\hline Perder - Ganar & $\begin{array}{l}0.398^{* *} \\
\text { Valor de } t(6.112)\end{array}$ & $\begin{array}{l}R^{2} \text { ajustada }=0.154 \\
\quad F=37.356 \\
\text { FIV = 1.000; Sig. } 0.000\end{array}$ \\
\hline Perder-Perder & $\begin{array}{l}0.630 * * \\
\text { Valor de t (11.419) } \\
\end{array}$ & $\begin{array}{c}R^{2} \text { ajustada }=0.394 \\
F=130.397 \\
\text { FIV = 1.000; Sig. } 0.000\end{array}$ \\
\hline Ganar-Ganar & $\begin{array}{c}0.081 \\
\text { Sin Valor de t }\end{array}$ & Sin Valor \\
\hline $\begin{array}{l}\text { Primero entender, luego ser } \\
\text { entendido }\end{array}$ & $\begin{array}{c}0.598^{* *} \\
\text { Valor de t (10.492) }\end{array}$ & $\begin{array}{c}R^{2} \text { ajustada }=0.354 \\
\quad F=110.089 \\
\text { FIV = 1.000; Sig. } 0.000\end{array}$ \\
\hline Reflexivo & $\begin{array}{c}0.254^{* *} \\
\text { Valor de t (3.694) } \\
\end{array}$ & $\begin{array}{c}R^{2} \text { ajustada }=0.060 \\
\quad F=13.643 \\
\text { FIV = 1.000; Sig. } 0.000\end{array}$ \\
\hline Independiente & $\begin{array}{c}(-) 0.051 \\
\text { Sin Valor de t }\end{array}$ & Sin Valor \\
\hline Planeador & $\begin{array}{c}0.161^{* *} \\
\text { Valor de t (2.299) }\end{array}$ & $\begin{array}{c}R^{2} \text { ajustada }=0.021 \\
F=5.286 \\
\text { FIV = 1.000; Sig. } 0.023\end{array}$ \\
\hline Analista & $\begin{array}{c}0.063 \\
\text { Sin Valor de t }\end{array}$ & Sin Valor \\
\hline Afilar la sierra & $\begin{array}{c}0.357^{* *} \\
\text { Valor de t (5.381) }\end{array}$ & $\begin{array}{c}R^{2} \text { ajustada }=0.123 \\
\quad F=28.958 \\
\text { FIV = 1.000; Sig. } 0.000\end{array}$ \\
\hline
\end{tabular}

Fuente: elaboración propia a partir de los datos obtenidos del SPSS v21. 
Los resultados de la Tabla 5 muestran que las dimensiones «Prioritario» y "Ganar-ganar» permiten suponer que ante la percepción de los empresarios, los empleados tendrán serias dificultades para fortalecer estas dimensiones, ya que acorde a la propuesta de Covey (2012), para que las personas puedan tener fortaleza en este tipo de dimensiones, es importante tener una disciplina fortalecida, además de tener equipos sinérgicos con liderazgo que les permita a los empleados tener sana competencia entre ellos, con la finalidad de mejorar la calidad de sus actividades laborales en este tipo de empresas manufactureras. Así mismo, se realizó la prueba de correlación de Pearson con el propósito de analizar la relación que tiene cada factor con la variable dependiente en donde los valores de t robusta, FIV, R cuadrada ajustada y valor de F permiten darle significancia a la relación entre cada dimensión y la variable dependiente. Los datos obtenidos se muestran en la Tabla 6.

En la Tabla 6 se puede observar que en la relación de las dimensiones del modelo con la variable dependiente, en particular las dimensiones: Tener un fin en mente, Prioritario, Ganar-ganar, Independiente y Analista, el análisis de correlación no tiene significancia o sentido como para poder afirmar que estas relaciones inciden de manera significativa en la aceptación por parte de los trabajadores en adoptar en sus actividades laborales la filosofía japonesa 9's. Por otro lado, las dimensiones que sí tienen impacto en la aceptación de una filosofía como las 9's son Perder - Perder; primero entender y luego ser entendido. Esto significa que, en el modelo teórico propuesto para estas empresas, y especulando temas de cultura, si bien como se mencionó en los análisis descriptivos existe interés por parte de los trabajadores de ser más sinérgicos, colaboradores y más proactivos, en realidad tienen alta tendencia a ser más individualistas, no colaboradores, así como mayormente centrados en sus propias necesidades más que en las de la empresa.

En la Tabla 7 se muestran las ecuaciones obtenidas del análisis de regresión donde se analizó cada dimensión con su variable dependiente.

Los resultados de la Tabla 7 muestran que no existe una relación de impacto de las dimensiones (Tener un fin en mente, Prioritario, Ganar-ganar, Independiente y Analista) con la variable dependiente (Aceptación de la filosofía japonesa 9's en el área de trabajo), lo que significa que estas relaciones no son significativas para este modelo aplicado a esta muestra con este objeto de estudio en la pyme manufacturera de Aguascalientes. En la Tabla 8 se muestran las ecuaciones derivadas de la relación de cada dimensión del bloque de "Hábitos» con la variable dependiente identificada como «Aceptación de la filosofía japonesa 9's en el área de trabajo».

Los resultados expresados en la Tabla 8, muestran que no existe relación significativa de las dimensiones: Tener un fin en mente, Prioritario, Ganar-ganar, Independiente y Analista con la variable dependiente de «Aceptación de la filosofía japonesa 9's en el área de trabajo», lo que significa que para la muestra en estas empresas, este tipo de dimensiones no son de impacto para poder afirmar que en el desarrollo de hábitos por parte de los trabajadores de estas organizaciones, estas dimensiones no inciden de manera significativa en la aceptación de una filosofía oriental como las 9's. Por otro lado, para los gerentes o encargados de las áreas productivas, estos resultados les permiten conocer que por cada unidad que se incremente, la variable dependiente se verá influenciada por la proporción mostrada en el valor de $\beta x$ descrita en cada ecuación. 
Tabla 7. Resumen del análisis del modelo de regresión

Table 7 . Summary of the analysis of the regression model

\begin{tabular}{|c|c|c|c|c|c|}
\hline \multirow[t]{2}{*}{ Variable Predictora } & \multicolumn{5}{|c|}{$\begin{array}{c}\text { Variable dependiente } \\
\text { (Aceptación de la filosofía japonesa 9's en el área de trabajo) }\end{array}$} \\
\hline & $\mathrm{R}$ & $R^{2}$ & $\mathrm{R}^{2}$ ajustada & $\begin{array}{l}\text { Error Típico de } \\
\text { estimación }\end{array}$ & Durbin - Watson \\
\hline Proactivo & $0.139^{a}$ & 0.019 & 0.014 & 0.852 & 1.042 \\
\hline Reactivo & $0.497^{a}$ & 0.247 & 0.243 & 0.747 & 1.357 \\
\hline Tener un fin en mente & \multicolumn{5}{|c|}{ Sin relación } \\
\hline Moroso & $0.504^{a}$ & 0.254 & 0.250 & 0.743 & 1.287 \\
\hline Prioritario & \multicolumn{5}{|c|}{ Sin relación } \\
\hline Sumiso & $0.547^{a}$ & 0.300 & 0.296 & 0.720 & 1.367 \\
\hline Flojo & $0.547^{a}$ & 0.299 & 0.295 & 0.720 & 1.242 \\
\hline Ganar-Perder & $0.424^{\mathrm{a}}$ & 0.181 & 0.176 & 0.779 & 1.143 \\
\hline Perder-Ganar & $0.398^{a}$ & 0.159 & 0.154 & 0.789 & 1.257 \\
\hline Perder-Perder & $0.630^{a}$ & 0.397 & 0.394 & 0.668 & 1.343 \\
\hline Ganar-Ganar & \multicolumn{5}{|c|}{ Sin relación } \\
\hline $\begin{array}{l}\text { Primero entender, luego } \\
\text { ser entendido }\end{array}$ & $0.598^{a}$ & 0.357 & 0.354 & 0.690 & 1.283 \\
\hline Reflexivo & $0.254^{a}$ & 0.064 & 0.060 & 0.832 & 1.160 \\
\hline Independiente & \multicolumn{5}{|c|}{ Sin relación } \\
\hline Planeador & $0.161^{a}$ & 0.026 & 0.021 & 0.849 & 1.044 \\
\hline Analista & \multicolumn{5}{|c|}{ Sin relación } \\
\hline Afilar la sierra & $0.357^{a}$ & 0.128 & 0.123 & 0.804 & 1.184 \\
\hline
\end{tabular}

Fuente: elaboración propia a partir de los datos obtenidos del SPSS v21.

Tabla 8. Resumen de ecuaciones de regresión

Table 8. Summary of regression equations

\begin{tabular}{|c|c|c|}
\hline Variable Dependiente & No. & Variable Independiente \\
\hline \multirow{17}{*}{$\begin{array}{c}\text { Aceptación de la filosofía } \\
\text { japonesa 9's en el área de } \\
\text { trabajo }\end{array}$} & 1 & $3.397-0.170$ Proactivo -0.139 \\
\hline & 2 & $1.535+0.451$ Reactivo +0.497 \\
\hline & 3 & Sin relación \\
\hline & 4 & $1.830+0.355$ Moroso +0.043 \\
\hline & 5 & Sin relación \\
\hline & 6 & $1.485+0.459$ Sumiso +0.050 \\
\hline & 7 & $1.791+0.375$ Flojo + 0.041 \\
\hline & 8 & $1.498+0.412$ Ganar-Perder + 0.063 \\
\hline & 9 & $1.590+0.392$ Perder-Ganar + 0.064 \\
\hline & 10 & $1.649+0.449$ Perder-Perder +0.039 \\
\hline & 11 & Sin relación \\
\hline & 12 & 1.6590 .436 Primero entender luego ser entendido +0.042 \\
\hline & 13 & $1.613+0.326$ Reflexivo +0.088 \\
\hline & 14 & Sin relación \\
\hline & 15 & $2.156+0.168$ Planeador +0.073 \\
\hline & 16 & Sin relación \\
\hline & 17 & $1.196+0.467$ Afilar la sierra +0.087 \\
\hline
\end{tabular}




\section{CONCLUSIONES}

En particular, en las empresas manufactureras por su naturaleza operativa, hoy en día es importante que los trabajadores tengan un rendimiento óptimo en las tareas que les sean encomendadas, ya que la exigencia del mercado es cada vez más fuerte. En este sentido, los trabajadores al margen de las políticas de las empresas y de las metodologías que utilicen de manera interna, sobre todo para el sector manufacturero donde se conoce que las reglas de trabajo son especiales por lo importante que es trabajar con tiempos justos, evitar demoras productivas y tener procesos donde no sean posibles las fallas, deben tener claro que para cumplir con sus tareas, debe existir mejora continua, desarrollo de sus habilidades y mayor participación en proponer estrategias que permitan a la empresa cumplir con los compromisos establecidos previamente con los clientes.

Y para ello, es muy necesario que dentro de este tipo de empresas, tanto gerentes como trabajadores consideren el cultivar el propósito de generar de manera constante acorde a sus capacidades personales el desarrollo de hábitos (Covey, 2012). El estudio muestra que para la aceptación por parte de los trabajadores de una filosofía de origen oriental (japonesa) como las 9's considerando como punto clave el desarrollo de hábitos por parte de los empleados, requiere de voluntad, convencimiento hacia los trabajadores por parte de los gerentes sobre la utilidad y beneficios de integrar esta filosofía en sus actividades de trabajo y de tiempo para su implementación.

Así mismo, los resultados estadísticos dejan en claro cuál es el mejor escenario que pretenden operar los gerentes al tener personal proactivo y sinérgico, ya que para este tipo de empresas, el trabajo en equipo y contar con personal que desarrolle hábitos e iniciativas propositivas, con este tipo de atributos personales, los empleados pueden lograr resultados competitivos para que las empresas sean más rentables, confiables y productivas. Sin embargo, también los resultados dejan en claro que, por entorno sociocultural, los trabajadores si bien cumplen con las tareas encomendadas en su jornada de trabajo, tienen altas tendencias en dejar fuera de su percepción personal el trabajar fuertemente en mejorar las prioridades, tener planes de mejora, fomentar el esquema de Ganar-ganar, ser más Independiente en donde se pueda tener la libertad de proponer mejoras significativas y ser más Analista.

En términos generales y al margen de la fase de experimentación en la cual se encuentra el modelo teórico, así como del instrumento de medición, no puede evitar ser considerado el hecho de que los aspectos culturales y sociales de los trabajadores están influenciados en sus rutinas y costumbres personales y esto hace que sean parte de su estilo de trabajo. Los resultados del presente estudio permiten considerar que para la adopción de cualquier herramienta de trabajo y en particular de la filosofía japonesa de 9's, es importante que los trabajadores de la pyme manufacturera de Aguascalientes tomen en consideración las siguientes recomendaciones:

- Ser proactivo, es decir, tener siempre una agenda con actividades enfocadas en un propósito y no solamente tener actividades por hacer ya que esto va a permitir en el incumplimiento con los compromisos adquiridos por no saber decir «no».

- Fortalecer la estrategia de Ganar-ganar, esto va a permitir mayor confiabilidad, más productividad y mejor comunicación para el desarrollo de proyectos y de trabajo en equipo. 
- Saber manejar las prioridades, esto significa que todo será importante sin embargo se requiere de habilidades y capacidades para clasificar emergencias.

- Tener mayor iniciativa, esto será posible si se tiene independencia en los empleados, no quiere decir que trabajen sin control o sin responsabilidades, sino que se sientan libres de opinar y de proponer mejoras a los procesos o porque no, en innovar.

- Ser más analistas, esto quiere decir que los trabajadores sin importar su nivel organizacional, respecto en cómo abordar las mejoras de sus procesos, requieren primeramente de conocer para poder diagnosticar y posteriormente tener la capacidad de aplicar técnicas de mejora.

Como limitaciones del estudio, es importante resaltar que, para la aplicación de este tipo de estudios, en las empresas se requiere de convencimiento y perseverancia para solicitar autorización especial con los dueños o gerentes para que permitan aplicar el instrumento de medición mismo que al margen de su fase experimental, es relevante que las empresas colaboradoras tengan más de 25 empleados para poder llevar a cabo la investigación. Como futuras líneas de investigación, se considera en ampliar la muestra, considerar otros sectores y realizar actividades simultáneas en lugares diversos, incluyendo traspasar las fronteras con el propósito de realizar análisis comparativos con mismo tipo de empresas.

\section{REFERENCIAS}

Aguilera, E. L.; González, A. M., \& Hernández, C. O. (2013). Impacto de la adopción de la filosofía japonesa 9's en la calidad del sector empresarial pyme en
Aguascalientes: un estudio empírico. México: Gasca.

Aymerich, L., Delgado, M., y Prado, E. (2010). Actitud y motivaciones hacia la publicidad interactiva en televisión. Questiones publicitarias, 15, 1-18.

Baltazar, G. G. (2005). Calidad: Pulcritud en 9 pasos. México, Revista Manufactura.

Beuren, I. M., \& Macohon, E. R. (2011). Institucionalização de hábitos e rotinas na contabilidade gerencial à luz da teoria da contingencia Um estudo em indústrias de móveis em São Bento do Sul. Revista Portuguesa e Brasileia de Gestao, 1(1), 7891.

Brown, D. H. \& Lockett, N. (2004). Potential of critical e-applications for engaging SMEs in e-business: a provider perspective. European Journal of Information Systems, 13(1), 21-34.

Caez, R. G. R., \& Casas, F. N. (2007). Formar en un estilo de vida saludable: otro reto para a ingeniería y la industria. Educación y Educadores, 10(2), 103-117.

Cantú, H. (2006). Desarrollo de una cultura de calidad. (3a ed.). México: Mc Graw Hill.

Covey, S. R. (1998). Los 7 hábitos de la gente altamente efectiva. New York: Paidós.

Cuéllar, Z. (2007). Proyecto 052/04 de reforma de la Ley 100 de 1993. Academia Nacional de Medicina de Colombia.

Dichter, E. (1960). La estrategia del deseo. Handbook of consumer motivation. New York: Mc Graw Hill.

De Anda, B. J., \& Rosales, H. O. (2009). Kaizen basado en 9's aplicado en el Instituto 
Tecnológico de Aguascalientes. Conciencia Tecnológica, 38(1), 35-38.

Deming, E. (1989). Calidad, productividad y competitividad. España: Editorial Díaz de Santos, S. A.

Deming, E. W. (1982). Quality productivity and competitivity positions. USA: Cambridge MIT.

Duhigg, Ch. (2012). The power of habit: Why we do what we do in life and business. Random House.

Frías, N. D. (2014). Apuntes de SPSS: análisis de fiabilidad de las puntuaciones de un instrumento de medida. Alfa de Cronbach: Un coeficiente de fiabilidad. España: Universidad de Valencia.

George, D., \& Mallery, P. (2003). SPSS for Windows step by step: A simple guide and reference. 11.0 update (4thed.). Boston: Allyn and Bacon.

Guedes, S. N. R. (2000). Observações sobre a economia institucional: há possibilidade de convergência entre o velho e o novo institucionalismo? Recuperado de www.race.nuca.ie.ufrj.br

Guerreiro, R., Frezatti, F., \& Casado, T. (2004). Em busca do entendimento da formação dos hábitos, rotinas $e$ instituições da contabilidade gerencia. In Congresso USP de Controladoria e Contabilidade, 4., Anais, FEA/USP, São Paulo.

Hernández, C. O. (2007). Desarrollo de un modelo de control administrativo en base a la filosofía japonesa 9's para las organizaciones en general. México: Universidad Autónoma de Aguascalientes.
Hernández, C.O., Colín, S.M., \& Galindo, R. O. A. (2015). La integración de 9's en Nivel de Calidad de los trabajadores de la pyme manufacturera: herramienta clave para la competitividad. México: Red Internacional de Investigadores en Competitividad.

Hernández, C.O., Colín, S.M., \& Galindo, R. O. A. (2016). La filosofía japonesa 9's: una herramienta clave para la competitividad de la pyme manufacturera. México: Red Internacional de Investigadores en Competitividad.

Hernández, C. O., López, Ch. M., \& Macías, H. J. H. (2012). Adopción de la filosofía 9's: ¿Una opción para la competitividad? España: Editorial Académica Española.

Hirano, H. (2000). 5 pilares de la fábrica visual. Japan: TGP Hoshin.

Hodgson, G. M. (2007). The revival of veblenian institutional economics. Journal of Economics Issues, 41(2), 325-340.

Imai, M. (2000). Cómo implementar el Kaizen en el sitio de trabajo (Genba). Japan: Mc. Graw Hill.

Instituto Nacional de Estadística y Geografía (INEGI). (2015). Directorio Estadístico Nacional de Unidades Económicas (DENUE). Recuperado de http://www3.inegi.org.mx/sistemas/map a/denue/default.aspx

Kume, H. (1989). Técnicas y herramientas para el mejoramiento de la calidad. México: Editorial Prentice May Hispanoamericana.

Lorenz, E. (2000). Organizational routines in the light of 'old' evolutionary economics: bringing politics back into the study of organizational learning. European Journal 
of Economic and Social Systems, 14(2), 191-207.

Mc Phail, F. E. (2012). Publicidad, hábitos y motivaciones. Revista Razón y Palabra, 17(80), 3-25.

Morgan, K. (1997). The learning region: Institutions, innovations and regional renewal, regional studies. The Journal of Region Studies Association, 31(5), 491503.

Nunnally, J. C. (1967). Psychometric theory. New York: McGraw Hill.

Oliver, C. (1997), «Sustainable competitive advantage: combining institutional and resource-based views». Strategic Management Journal, 18(9), 697-713.

Ortiz, G., Esquivel, E. O., \& Hernández, O. (2016). El impacto de la relación con el cliente y de la capacidad de valor agregado en el servicio en el rendimiento de la pyme manufacturera de Aguascalientes. Revista CEA, 2(4), 47-5

Pérez, E. S. (2011). 5 y 9 S's Beneficios e Implantación. México: Instituto Tecnológico Superior de Calkiní en el Estado de Campeche.

Prins, N. M. A., \& Gómez, L. M. J. (2015). Índice para la medición de la competitividad en Colombia. Revista CEA, 1(2), 109-121

Scapens, R. W. (1994). Never mind the gap: towards an institutional perspective on management accounting practice. Management Accounting Research, 5(3/4), 301-321.

Vargas, R. H. (2004). Manual de implementación de las 5's de la Corporación Autónoma Regional de Santander, versión 1.0. España. 
Análisis de la relación hábitos y aceptación de 9's como elemento de competitividad en la pyme manufacturera de Aguascalientes, México 